\title{
Chondrocyte viability depends on the preservative solution
}

\author{
Krzysztof Gawęda ${ }^{1 *}$, Marta Tarczyńska ${ }^{1}$, Ewa Olender ${ }^{2}$, Izabela Uhrynowska-Tyszkiewicz ${ }^{2,3}$, \\ Artur Kamiński ${ }^{2,3}$

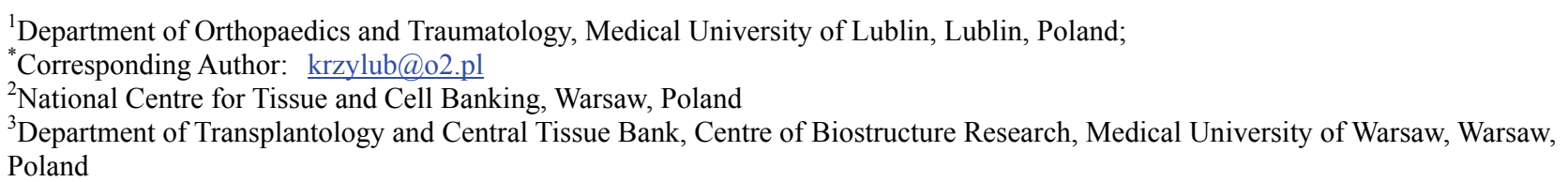

Received 11 February 2010; revised 15 March 2010; accepted 18 March 2010.

\begin{abstract}
Fresh osteochondral grafts find broad application in the treatment of extensive and focal damages of joint surfaces. The maintenance of chondrocyte viability of the collected grafts is of key importance. Aim: The evaluation of chondrocyte survivability in a stable temperature of $4^{\circ} \mathrm{C}$ in different preservative solutions is the aim of this work. Method: Chondrocyte survivability has been evaluated in saline solution (group I), Ringer solution (group II), saline with an addition of hyaluronic acid (group III) and saline enriched with glucosamine sulphate (group IV). The amount of live chondrocytes was examined on the day of collection and subsequently after 1, 2, 3,6,12, and 21 days using the Promega MTT test. Results: The highest number of live chondrocytes as calculated for $1 \mathrm{~g}$ of hyaline cartilage after 21 days was ascertained in group IV (saline with glucosamine sulphate). The lowest number of live chondral cells was observed in group II (saline with hyaluronic acid). Chondrocyte survivability in saline (group I) was higher than in the Ringer solution (group II). Conclusions: The enrichment of saline solution with glucosamine sulphate protracts the viability of chondrocytes in fresh osteochondral grafts prepared for chondral transplantation.
\end{abstract}

Keywords: Chondrocyte; Preservative Solution; Osteochondral Grafts; Fresh Grafts

\section{INTRODUCTION}

The use of fresh allogeneic osteochondral grafts to repair extensive damage to joint surfaces spans more than 100 years [1]. Grafts of many different types and sizes have been used, from various cylindrical osteochondral grafts with radii ranging from several millimeters to larger than $1 \mathrm{~cm}$, through shell grafts, to the transplantation of whole joint surfaces of tibial or femoral condyles [2]. The size of fresh allogeneic osteochondral grafts notwithstanding, the determination and maintenance of safe conditions for the donor joint surfaces remains an ongoing issue. It is crucial to maintain strict sanitary standards for grafts until their implantation. Although rules regarding such standards are determined by laws, and thus differ by country, it is important for hospitals to organize a specialized transplant service with procedural guidelines, which will mitigate difficulties encountered while performing this repair method. The tasks of such a service should include recruiting donors and preparing potential recipients for transplantation. Efficient use of fresh allogeneic grafts of joint surfaces is essential because the chondrocytes of transplanted cartilage atrophy rapidly. Tissue survival can be extended by cooling collected fragments. However, freezing typically destroys live chondral cells and disintegrates the intercellular matrix structure [3]. Thus, maintaining the viability of collected chondrocytes is a key issue to be addressed at centers that use this method of joint cartilage repair. Significant chondrocyte survival can be maintained for only 1-2 weeks by preserving osteochondral grafts in normal saline with antibiotics, buffered normal saline, or Ringer solution cooled to $4^{\circ} \mathrm{C}$, although this period can be extended [4-6]. Thus there is a continuing need for methods that prolong the safe preservation and transport of graft materials with live chondrocytes.

Pharmaceuticals and dietary supplements that purportedly improve chondrocyte vitality and the ability of damaged chondral surfaces to self-repair have gained 
popularity in recent years. Various derivatives of hyaluronic acid and substances containing glucosamine are most frequently used.

\section{AIM}

The widespread use of hyaluronic acid and glucosamine compounds in the pharmacotherapy of focal and generalized joint cartilage damages raises the prospect of possibly extending chondrocyte vitality by adding these substances to the transport solutions for osteochondral grafts. Therefore, in this study, we determined the survival of human chondral cells stored in vitro at the same temperature, but in different conserving solutions.

\section{MATERIALS AND METHODS}

The distal femoral epiphysis was collected along with the cartilage coating from six male donors, aged 21-48 years, in the operating room after the conclusion of multi-organ collections. The obtained osteochondral specimens were divided into four equal parts. Each was randomly assigned for immersion in one of the following four media: normal saline solution (group I), Ringer solution (group II), normal saline solution with $20 \mathrm{mg}$ sodium salt of hyaluronic acid (group III), or normal saline solution enriched with $1.5 \mathrm{~g}$ glucosamine sulphate (group IV). Each medium was enriched with $1.0 \mathrm{~g}$ thirdgeneration cephalosporin. Each of the obtained quarters of the distal femoral epiphysis was subsequently divided into seven equal parts, which were placed in separate sterile containers filled with the evaluated solution. All samples were stored at $4^{\circ} \mathrm{C}$. Live chondrocyte numbers in the initial samples of each group were quantitatively analyzed within $24 \mathrm{~h}$ after collection using colorimetric reduction in the Promega MTT (3-(4, 5-dimethylthiazol2-yl)2, 5-diphenyl tetrazolium bromide) test. Evaluations were subsequently conducted on Days 1, 2, 3, 6, 12, and 21 . The obtained absorption capacity was proportional to the cellular oxidoreductive activity and subsequently scaled to $1 \mathrm{~g}$ hyaline cartilage. Measurement results were evaluated using analysis of variance (ANOVA) and Friedman's and Dunnet's statistical tests.

The research was approved by the Bioethical Commission of the Medical University of Lublin (PL) no. KE-0254/143/2006, according to Good Clinical Practice conditions.

\section{RESULTS}

None of the evaluated groups showed a statistically significantly change in absorption from initial values during the first 3 days of observation. Group IV (saline solution enriched with glucosamine) had the highest absorption values, both initially and during the subsequent evaluation days, as calculated per gram of cartilage. A statistically significant decline in live chondrocyte numbers occurred between Days 3 and 6 of storage in Ringer fluid (group II) and saline with sodium salt of hyaluronic acid (group III). A similar decline occurred in normal saline (group I) between Days 12 and 21 of storage. No statistically significant decrease in the number of live chondrocytes was evident in group IV (saline enriched with glucosamine sulphate) over the entire observation period.

At Day 21, group IV samples had the highest number of live chondrocytes, and the difference in counts between this group and each other group was statistically significant.

The lowest numbers of live chondrocytes throughout the whole research period were found in group III, saline solution with sodium salt of hyaluronic acid. The most

Table 1. Absorbance values of Ringer's solution vs. other media.

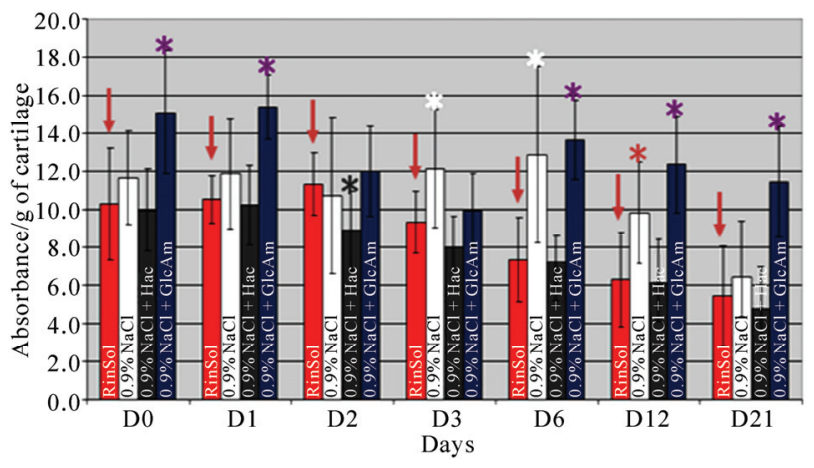

Chondrocyte viability was highest in the $0.9 \% \mathrm{NaCl}+$ glucosamine group on all observation days.

Table 2. Trend analysis of absorbance values.

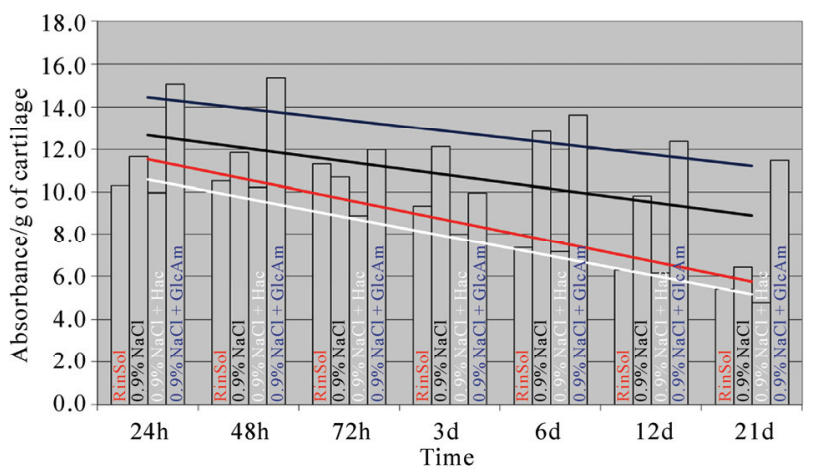

The lowest absorbance values and the biggest decrease in values were seen in group III $(0.9 \% \mathrm{NaCl}+\mathrm{HAc})$. The highest absorbance values and the smallest drop in values were seen in group IV $(0.9 \% \mathrm{NaCl}+$ glucosamine) 
rapid drops in numbers of live chondral cells were also observed in this group. The number of live chondral cells in group II (Ringer solution) was lower than in group I (normal saline solution).

\section{DISCUSSION}

The storage period of fresh osteochondral grafts greatly affects their clinical value, where longer storage reduces the number of live chondral cells [7].Allen et al. reported a significant decline in live chondral cell numbers in osteochondral grafts within 3 weeks of collection [4]. Previous research has focused on protecting chondrocyte vitality in allogeneic osteochondral grafts primarily by changing the temperature. Voss et al. demonstrated that storing cartilage at room temperature accelerated cell death [8], and a critical increase occurred between $50^{\circ} \mathrm{C}$ and $55^{\circ} \mathrm{C}$. In contrast, lowering the temperature is generally favorable to chondrocyte survival. Pearsall reported that the number of live chondrocytes dropped to $67 \%$ of the initial value after a storage period of 44 days at $4{ }^{\circ} \mathrm{C}$ [9]. Judas et al. showed that adding protective substances to the agents in which osteochondral grafts were stored before freezing extended chondrocyte survival [10]. Adding tetracycline-type antibiotics may improve the vitality of chondral graft cells [11]. Teng et al. examined the impact of IGF-1(insulin-like growth factor 1) and the apoptosis inhibitor zVAD-fmk in Ringer fluid and Dulbecco's modified Eagle's medium (DMEM) on the survival of bovine chondrocytes [7]. Chondrocytes atrophied most rapidly in Ringer fluid; the process was slower in DMEM. Adding either IGF-1 or zVAD-fmk to the DMEM significantly extended the survival of chondral cells. Pennock analyzed the survivability of human chondrocytes in osteochondral grafts suspended in normal saline, glucose, amino acid solution, and $10 \%$ fetal bovine serum solution [12]. Chondrocyte survival was significantly lower in solutions without bovine serum. However, storing tissue with bovine serum requires further research on the possibility of the transmission of infections as well as immune reactions to proteins from foreign species.

The results of our research demonstrate that chondrocyte cell death was quickest in saline solution enriched with sodium salt of hyaluronic acid. We are not in a position to determine why adding hyaluronic acid derivatives did not improve chondrocyte graft vitality. Samples of human joint cartilage stored for 3 weeks in saline solution enriched with glucosamine sulphate retained their vitality to the greatest degree compared to samples preserved in normal saline alone, Ringer solution, and normal saline solution plus sodium salt of hyaluronic acid. Thus, chondrocyte vitality can be increased by enriching the fluid environment of graft preservation with glucosamine sulphate. The lowest decline in live chondrocyte numbers over a 3-week period decreased the biological value of the graft only slightly. Our results show that the inevitable process of in vitro chondral cell atrophy during the storage period for surgical transplantation of joint surfaces may be slowed to maintain high biological and mechanical value of the graft. Otsuki et al. showed that a decrease in glycosaminoglycan concentration did not lead directly to the intensification of chondral joint cell atrophy [13]. Based on our analyses, increasing the amount may slow these processes.

\section{REFERENCES}

[1] Lexer, E. (1908) Substitution of whole or half joints from freshly amputated extremities by free plastic operations. Surgery, Gynecology and Obstetrics, 6, 601-607.

[2] Gross, A.E., Silverstein, E.A., Falk, J., Falk, R. and Langer, F. (1975) The allotransplantation of partial joints in the treatment of osteoarthritis of the knee. Clinical Orthopaedics and Related Research, 108, 7-14.

[3] Csönge, L., Bravo, D., Newman-Gage, H., Rigley, T., Conrad, E.U., Bakay, A., Strong, D.M. and Pellet, S. (2002) Banking of osteochondral allografts, Part II. Preservation of Chondrocyte Viability during Long-Term Storage. Cell and Tissue Banking, 3(3), 161-168.

[4] Allen, T.R., Robertson, C.M., Pennock, A.T., Bugbee, W.D., Harwood, F.L., Wong, W., Chen, A.C., Sah, R.L. and Amiel, D. (2005) Analysis of stored osteochondral allografts at the time of surgical implantation. America Journal of Sports Medicine, 33(10), 1479-1484.

[5] LaPrade, R.F., Botker, J., Herzog, M. and Agel, J. (2009) Refrigerated osteoarticular allografts to treat articular cartilage defects of the femoral condyles. Journal of Bone and Joint Surgery American, 91(4), 805-811.

[6] Williams III, R.J., Dreese, J.C., Chen and C.-T. (2004) Chondrocyte survival and material properties of hypothermically stored cartilage. An evaluation of tissue used for osteochondral allograft transplantation. American Journal of Sports Medicine, 32(1), 132-139.

[7] Teng, M.S., Yuen, A.S. and Kim, H.T. (2008) Enhancing osteochondral allograft viability: effects of storage media composition. Clinical Orthopaedics and Related Research, 466(8), 1804-1809.

[8] Voss, J.R., Lu, Y., Edwards, R.B., Bogdanske, J.J., Markel, M.M. (2006) Effects of thermal energy on chondrocyte viability. American Journal of Veterinary Research, 67(10), 1708-1712.

[9] Pearsall, A.W., Tucker, J.A., Hester, R.B. and Heitman, R.J. (2004) Chondrocyte viability in refrigerated osteochondral allografts used for transplantation within the knee. American Journal of Sports Medicine, 32(1), 125131.

[10] Judas, F., Rosa, S., Teixeira, L., Lopes, C. and Ferreira Mendes, A. (2007) Chondrocyte viability in fresh frozen large human osteochondral allografts: effect of cryoprotective agents. Transplantations Proceedings, 39(8), 2531-2534. 
[11] Snasshall, J.T., Teng, M.S., Yuen, A.S. and Kim, H.T. (2006) Minocycline increases articular chondrocyte viability during storage of osteochondral allografts. 52nd Annual Meeting of the Orthopedic Research Society, Chicago, paper 0267.

[12] Pennock, A.T., Wagner, F., Robertson, C.M., Harwood, F.L., Bugbee, W.D., Amiel, D. (2006) Prolonged storage of osteochondral allografts: Does the addition of fetal bovine erum improve chondrocyte viability? The Journal of Knee Surgery, 19, 265-272.

[13] Otsuki, S., Brinson, D.C., Creighton, L., Kinoshita, M., Sah, R.L., D'Lima, D., Lotz, M. (2008) The effect of glycosaminoglycan loss on chondrocyte viability: A study on porcine cartilage explants. Arthritis and Rheumatism, 58(4), 1076-1085. 